\title{
Probing Enantioselectivity with X-Ray Photoelectron Spectroscopy and Density Functional Theory
}

\author{
R. Schillinger, ${ }^{1,2}$ Ž. Šljivančanin, ${ }^{3}$ B. Hammer, ${ }^{4}$ and T. Greber ${ }^{1, *}$ \\ ${ }^{1}$ Physik Institut Universität Zürich, Winterthurerstrasse 190, CH-8057 Zürich, Switzerland \\ ${ }^{2}$ Swiss Light Source, Paul Scherrer Institut, CH-5232 Villigen, Switzerland \\ ${ }^{3}$ École Polytechnique Fédérale de Lausanne (EPFL), Institut Romand de Recherche Numérique en Physique des Matériaux (IRRMA), \\ CH-1015 Lausanne, Switzerland \\ ${ }^{4}$ Department of Physics and Astronomy and iNANO, University of Aarhus, DK-8000 Aarhus C, Denmark
}

(Received 12 September 2006; published 27 March 2007)

\begin{abstract}
The enantioselectivity of gold is investigated by x-ray photoelectron spectroscopy (XPS) and density functional theory (DFT). Cysteine molecules on a chiral Au(17 119$)^{S}$ surface show enantiospecific core level binding energies in the amino and in the thiol group. The sign and order of magnitude of the XPS core level shifts is reproduced by DFT. Identical preparations of $D$ - and $L$-cysteine layers lead to $D$-cysteine molecules in the pure $\mathrm{NH}_{2}$ form, while a small portion of $L$-cysteine molecules maintains a hydrogen rich amino group $\left(\mathrm{NH}_{3}\right)$. This implies enantiospecific adsorption reaction pathways and is consistent with DFT that indicates an activated hydrogen abstraction reaction from the amino group, which is downhill for $D$-cysteine.
\end{abstract}

DOI: 10.1103/PhysRevLett.98.136102

Molecular separation has an enormous technological importance. Beyond thermodynamic equilibrium considerations, the understanding of the underlying processes is far from being complete because it involves the tracking of molecules in space and time. It is, however, known that a difference in chemical potential between two molecular species is required for separation. For chiral molecules, where the two enantiomers have the same chemical potential, the process must involve enantioselectivity [1]. Lefthanded and right-handed molecules may be sorted out via the formation of molecular pairs, with different chemical potential which are split again after separation. Of course, the two molecules that form the pairs have to be different and not enantiomorphous if left $(L)$ shall be separated from right $(D)$ [2]. Enantiomeric separation of $L$ and $D$ requires chiral heterorecognition, i.e., the formation of diastereomers $(L, S)$ or $(D, S)$, where $S$ describes the handedness of the molecular unit which distinguishes $L$ from $D$. Using kinked surfaces, chiral heterorecognition has been established in extracting enantiospecific adsorption energy differences from experimental data [3,4]. Recently, chiral heterorecognition was concluded from enantiospecific adsorption geometries on gold surfaces with kinks [5,6]. For the case of a vicinal $\mathrm{Au}(111)$ surface, i.e., $\operatorname{Au}(17119)^{S}$, which has $S$ kinks only, it was demonstrated that the two enantiomorphs of cysteine adsorb in two distinct, nonmirror symmetric structures [5]. The Miller indices (17 119) determine the crystal cut direction and their sequence the chirality of the kinks, where, e.g., $\mathrm{Au}(17911)$ has the same miscut but $R$ kinks only [7,8]. Figure 1 shows the corresponding adsorption structures of $D$ - and $L$-cysteine on $\mathrm{Au}\left(\begin{array}{lll}17 & 11 & 9\end{array}\right)^{S}$. Inspection of the picture indicates different bonding of the two enantiomers from which a different bond energy is inferred. The amino bond strength of
PACS numbers: 68.43.Bc, 79.60.-i, 82.65.+r, 87.15.By

$D$-cysteine was, e.g., found to be about $0.19 \mathrm{eV}$ stronger than that of $L$-cysteine, which reflects the bonding to gold atoms with different coordination. In this context, core level spectroscopies, which are sensitive to the chemical identity and the environment of an atom, are perfect tools to probe differences in bond structure at the atomic level [9]. Here we further investigate the cysteine/ $\mathrm{Au}(17119)^{\mathrm{S}}$ system and show spectroscopic evidence for chiral hetero-

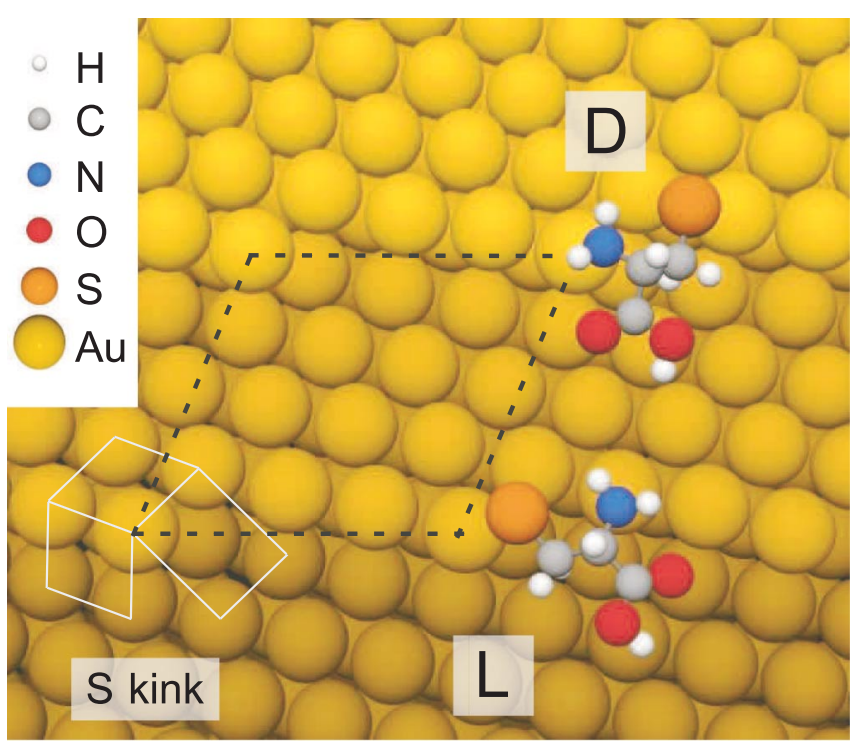

FIG. 1 (color online). Structural model of cysteine on $\mathrm{Au}(17119)^{S}$ from [5]. The unit cell of the (17 11 9) surface is marked with dashed lines. In the case of $L$-cysteine the amino group binds to a step atom, while for $D$-cysteine it binds to the kink atom. The anticlockwise sequence of $\{110\},\{111\},\{100\}$ facets defines a $S$ kink. The color code of the atoms is given on the left. 
recognition, where core levels of $D$ - and $L$-cysteine have distinct binding energies. This independently confirms that the two enantiomers of cysteine bind differently on a $\mathrm{Au}\left(\begin{array}{lll}17 & 11 & 9\end{array}\right)^{S}$ surface. Furthermore, there are significant enantiospecific differences in the hydrogen abstraction from the amino group of cysteine. This indicates the presence of enantiospecific reaction pathways which is in full agreement with density functional theory (DFT) calcula-

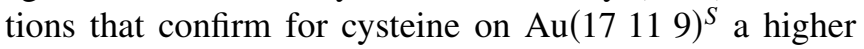
hydrogen affinity to the amino group of $L$-cysteine than to that of $D$-cysteine.

The experiments were carried out at the X11MA beam line of the Swiss Light Source in an end station for X-ray photoelectron spectroscopy (XPS) and angle scanned x-ray photoelectron diffraction (XPD) $[5,10]$. The base pressure in the system was below $10^{-10}$ mbar. The gold substrate (MaTecK) was cleaned by sputter and annealing cycles. Gentle annealing to $400{ }^{\circ} \mathrm{C}$ resulted in a clear (17 119) LEED pattern. $D$-cysteine (Fluka; 99\%) and $L$-cysteine (Sigma; 98\%) were evaporated from a dual Knudsen-cell evaporator. Prior to experiments the loaded evaporator was thoroughly degassed at $125^{\circ} \mathrm{C}$, resulting in a total pressure of less than $5 \times 10^{-9} \mathrm{mbar}$. Deposition of cysteine at room temperature and short annealing to $\approx 350 \mathrm{~K}$ lead to a coverage of $0.8 \pm 0.1$ molecules per kink site. Several experimental runs with the same reproducible preparation procedure were performed for both enantiomers. In each run we recorded a time series of gold, nitrogen, and sulfur spectra. In all photoemission experiments light with linear horizontal polarization and a photon energy of $770 \mathrm{eV}$ was used. The overall electron energy resolution was $1 \mathrm{eV}$ full width at half maximum (FWHM). The electron energies were consistently calibrated by the position of the Fermi level, the $\mathrm{Au} 4 f_{7 / 2}(84.0 \mathrm{eV})$ and the $\mathrm{Au} 4 d_{5 / 2}(353.1 \mathrm{eV})$ core levels. In order to enhance the surface sensitivity the spectra were recorded at grazing emission angles $\left(76^{\circ}\right.$ from the surface normal). The XPS data of the $\mathrm{N} 1 s, \mathrm{~S}$ $2 p$, and the $\mathrm{O} 1 s$ core levels indicate cysteine predominantly in the $\mathrm{SC}_{\beta} \mathrm{H}_{2}-\mathrm{C}_{\alpha} \mathrm{H}-\mathrm{NH}_{2}-\mathrm{COOH}$ state. All $D$-cysteine preparations contain $\mathrm{NH}_{2}$ amino groups only. On the other hand, all $L$-cysteine preparations show some $\mathrm{NH}_{3}$ admixture, which appears to be sensitive to $\mathrm{x}$-ray exposure, as it was also found in XPS investigations of thick cysteine layers [11]. Under X-ray exposure the $\mathrm{NH}_{3}$ component in the $\mathrm{N} 1 s$ spectrum decreases exponentially and the $\mathrm{NH}_{2}$ component increases correspondingly. From the exponential decay constant of the $L$-cysteine $\mathrm{NH}_{3}$ species and the secondary electron emission current density of $\approx 5 \mathrm{~mA} / \mathrm{m}^{2}$ we get an overall cross section for hydrogen abstraction of $L$-cysteine $\mathrm{NH}_{3}$ in the order of $\approx 10^{-20} \mathrm{~m}^{2}$.

Using density functional theory (DFT) and ultrasoft pseudopotentials $[12,13]$ as implemented in the DACAPO package $[14,15]$, we calculated the difference in the $\mathrm{N} 1 s$ core level binding energies of $L$ - and $D$-cysteine adsorbed on the $\mathrm{Au}(17119)$ surface. The adsorption geometries are taken from our previous study [5]. The enantiomeric core level binding-energy shifts are computed following the procedure described in Refs. [16,17]. For each enantiomer, two separate calculations were performed. We first calculated the uncharged electronic ground state, and then replaced the N (S) pseudopotential by another pseudopotential, with a $1 s(2 p)$ hole present in the core and repeated the calculation. Thereafter, we calculated the total energy differences of the ground state and of the state with a $1 s(2 p)$ core hole. The enantiomeric core level shift is obtained by comparing the corresponding total energy differences of $L$ - and $D$-cysteine on $\mathrm{Au}(17119)$, respectively. The activation energies for the $\mathrm{NH}_{3}$ hydrogen abstraction were determined performing geometry optimization calculations, where the $\mathrm{N}-\mathrm{H}$ bond length of the hydrogen atom which is abstracted was incrementally increased from 1.5 to $2.0 \AA$. Theory uses the same numbers of hydrogen atoms bound to the amino, the carboxyl, and the thiol group as found in the experiments. The polarization of the substrate and the molecule and the concomitant ionicity of the different cysteine subgroups is implicitly determined by the self-consistent optimization of the nuclear coordinates and the corresponding electron densities, which we do not quote, since they were not directly accessed by XPS.

The $\mathrm{N} 1 s$ binding energy of $D$-cysteine on $\mathrm{Au}\left(\begin{array}{llll}17 & 11 & 9\end{array}\right)^{S}$ is $399.6 \pm 0.1 \mathrm{eV}$ [Fig. 2(a)] $(\mathrm{FWHM}=1.1 \mathrm{eV})$. For $L$-cysteine the $\mathrm{N} 1 s$ binding energy is about $200 \mathrm{meV}$ lower, which compares well with the cysteine $\mathrm{NH}_{2}$ binding energy on $\mathrm{Au}(110)$ of $399.4 \pm 0.2 \mathrm{eV} \mathrm{[18]} \mathrm{and} \mathrm{the} \mathrm{circum-}$ stance that the amino group binds to a sevenfold coordi-
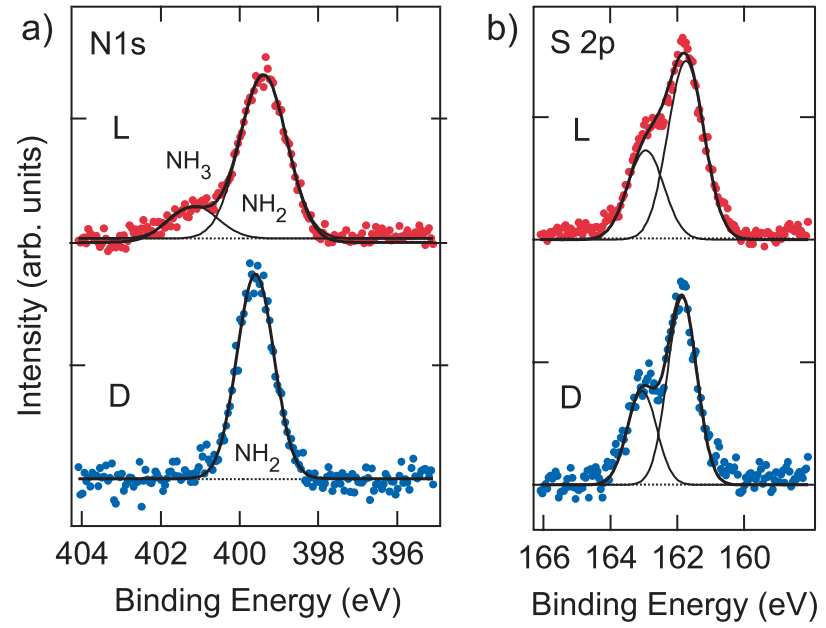

FIG. 2 (color online). X-ray photoelectron emission spectra for $D$ - (blue) and $L$-cysteine (red) on $\mathrm{Au}\left(\begin{array}{lll}17 & 11 & 9\end{array}\right)^{S}$. (a) $\mathrm{N} 1 s$ region. The constant background and two or one Gaussians to fit the $L$ or $D$-cysteine spectra, respectively, were indicated with lines. The $\mathrm{NH}_{2}$ components dominate, where in the case of $L$-cysteine also a $\mathrm{NH}_{3}$ component is visible as a high binding-energy shoulder. (b) $\mathrm{S} 2 p$ region. The spectra are fitted with a doublet of Gaussians with a fixed spin-orbit splitting of $1.2 \mathrm{eV}$ and an intensity ratio of 1:2. The fits are solid black lines. 
nated gold atom [19]. The core level binding-energy difference between adsorbed $D$-cysteine and $L$-cysteine is an enantiospecific core level shift and indicates chiral recognition of cysteine by $\mathrm{Au}\left(\begin{array}{lll}17 & 11 & 9\end{array}\right)^{S}$. It can be rationalized by enantiospecific bonding to a kink or a step atom (see Fig. 1). Furthermore, the $L$-cysteine $\mathrm{N} 1 s$ spectrum is broader and has a second amino component, which is identified as $\mathrm{NH}_{3}$ species at $1.9 \mathrm{eV}$ higher binding energy $[18,20-22]$. This is very interesting on its own (see below); it does, however, also bear the source of systematic errors, which have to be known when the observed shifts shall be compared with theory. Since the $L$-cysteine $\mathrm{NH}_{3}$ weight decreases as the $L$-cysteine $\mathrm{NH}_{2}$ weight increases under Xray irradiation, we were able to track the influence of $\mathrm{NH}_{3}$ hydrogen abstraction on the $\mathrm{NH}_{2}$ binding-energy shift. The thiol groups also have different bonding sites for $D$-cysteine or $L$-cysteine. Figure 2 (b) shows an enantiospecific core level shift of $\approx 100 \mathrm{meV}$ for the $\mathrm{S} 2 p$ level, although the adsorption geometries have similar bridge sites for the sulfur bonding in both cases (see Fig. 1) [23]. As for the case of $\mathrm{N} 1 s$, the S $2 p$ core level binding energy of $D$-cysteine remains constant $(161.9 \pm 0.1 \mathrm{eV})$ and is close to the $\mathrm{S} 2 p$ binding energy of cysteine on $\mathrm{Au}(110)$ of $161.95 \pm 0.05 \mathrm{eV}$ [18]. For $L$-cysteine it decreases with $\mathrm{x}$-ray exposure time. Apparently the $\mathrm{NH}_{2}: \mathrm{NH}_{3}$ composition related shifts are stronger for sulfur than for nitrogen. This points to a change in sulfur bonding site in going from $\mathrm{NH}_{3}$ to $\mathrm{NH}_{2}$, while the $\mathrm{N} 1 s$ shift for the $\mathrm{NH}_{2}$ nitrogen may solely be related to the influence of the spectral weight of the $\mathrm{NH}_{3}$ shoulder on the $\mathrm{NH}_{2}$ energy position.

In Fig. 3 the decrease of the $L$-cysteine $\mathrm{NH}_{3}$ weight and the core level shifts were shown as a function of $\mathrm{x}$-ray exposure time. They give a natural explanation for the broader $L$-cysteine core levels. Using the decay constant of the $L$-cysteine $\mathrm{NH}_{3}$ weight for the determination of $\Delta E_{B}(\mathrm{~S} 2 p)_{t \rightarrow \infty}$ we find a significant shift of $145 \pm$ $50 \mathrm{meV}$, while $\Delta E_{B}(\mathrm{~S} 2 p)_{t \rightarrow 0}$ is almost zero ( $-40 \pm$ $30 \mathrm{meV}$ ). Comparing the experimentally determined enantiospecific core level shifts with the theoretical results, which base on the published structures for $D$ - and $L$-cysteine [5], we find a better agreement with the $t \rightarrow$ $\infty$ extrapolated values (see Table I). In particular, the XPS data indicate a higher hydrogen affinity of the amino group in the $L$-cysteine $/ \mathrm{Au}\left(\begin{array}{lll}17 & 11 & 9\end{array}\right)^{S}$ configuration, i.e., enantiospecific reactivity.

In order to further substantiate the aspect of enantiospecific reaction pathways, we calculated the cysteine $\mathrm{CY}-\mathrm{NH}_{3} \rightarrow \mathrm{CY}-\mathrm{NH}_{2}+1 / 2 \mathrm{H}_{2}(g)$ potential energy surfaces of adsorbed $L$ - and $D$-cysteine $(\mathrm{CY}=$ $\left.\mathrm{SC}_{\beta} \mathrm{H}_{2}-\mathrm{C}_{\alpha} \mathrm{H}-\mathrm{COOH}\right)$. The most important results are summarized in Fig. 4. For both enantiomers the reaction is activated, where the transition state for $L$-cysteine is higher than that for $D$-cysteine. It also turns out that there is no net energy gain upon hydrogen abstraction from $\mathrm{NH}_{3}$ for $L$-cysteine. The apparent inconsistency with the experi-

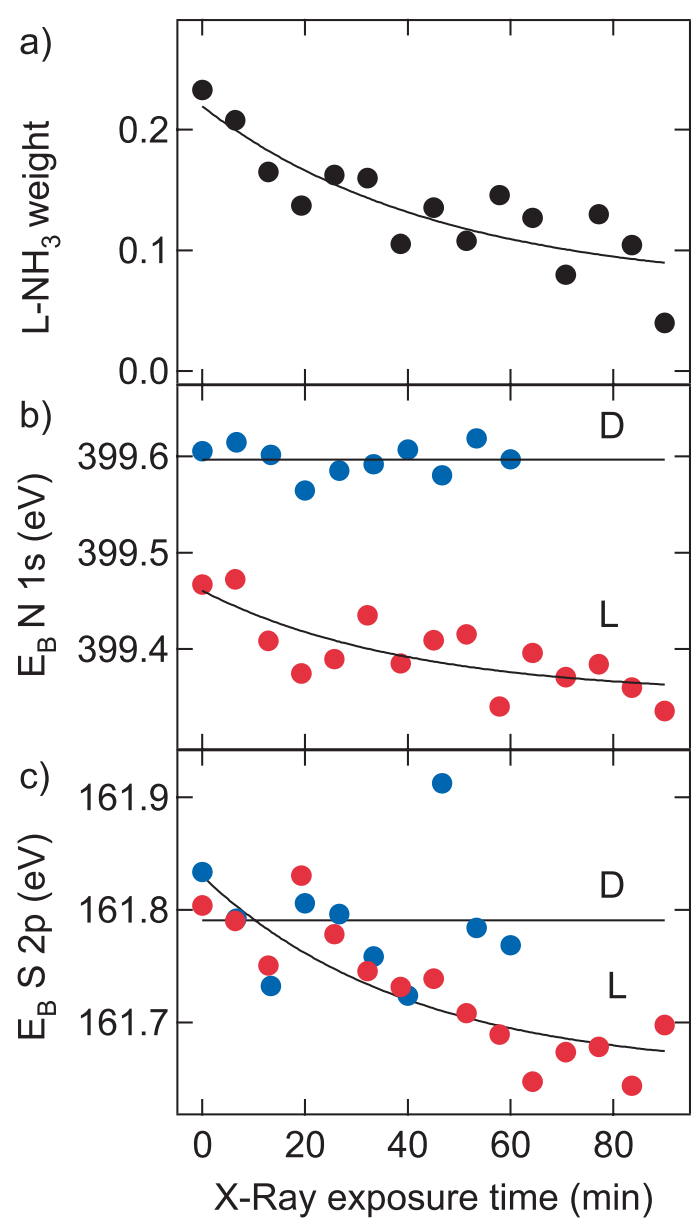

FIG. 3 (color online). Systematic alteration of the core level spectra with $\mathrm{x}$-ray irradiation time. (a) Decrease of the $L$-cysteine $\mathrm{NH}_{3}$ spectral weight. (b) $\mathrm{N} 1 s$ binding energies for $L$-cysteine (red circles) and $D$-cysteine (blue circles). (c) S $2 p$ binding energies for $L$-cysteine (red) and $D$-cysteine (blue circles). The fits (solid lines) in (b) and (c) adopt for $D$-cysteine a constant and for $L$-cysteine an exponential with the decay constant found from the $L$-cysteine $\mathrm{NH}_{3}$ spectral weight shown in (a).

ments that do not show a 1:1 equilibrium between $L$-cysteine $\mathrm{NH}_{3}$ and $L$-cysteine $\mathrm{NH}_{2}$ can be explained by the fact that the abstracted hydrogen may desorb into the gas phase from where it is pumped away, which accordingly shifts the equilibrium to the hydrogen poor side. For $D$-cysteine the hydrogen abstraction reaction is downhill

TABLE I. Experimental and theoretical enantiomeric core level shifts $\Delta E_{B}=E_{B}^{D}-E_{B}^{L}$ for $D$ - and $L$-cysteine adsorbed on $\mathrm{Au}\left(\begin{array}{lll}17 & 11 & 9\end{array}\right)^{S}$. The two experimental values correspond to the extrapolated values for x-ray exposure times $t \rightarrow 0$ and $t \rightarrow \infty$.

\begin{tabular}{lccc}
\hline \hline & \multicolumn{2}{c}{ Experiment $(\mathrm{meV})$} & Theory (meV) \\
Core level & $t \rightarrow 0$ & $t \rightarrow \infty$ & \\
\hline $\mathrm{N} 1 s$ & $141 \pm 20$ & $250 \pm 50$ & 360 \\
$\mathrm{~S} 2 p$ & $-40 \pm 30$ & $145 \pm 50$ & 83 \\
\hline \hline
\end{tabular}




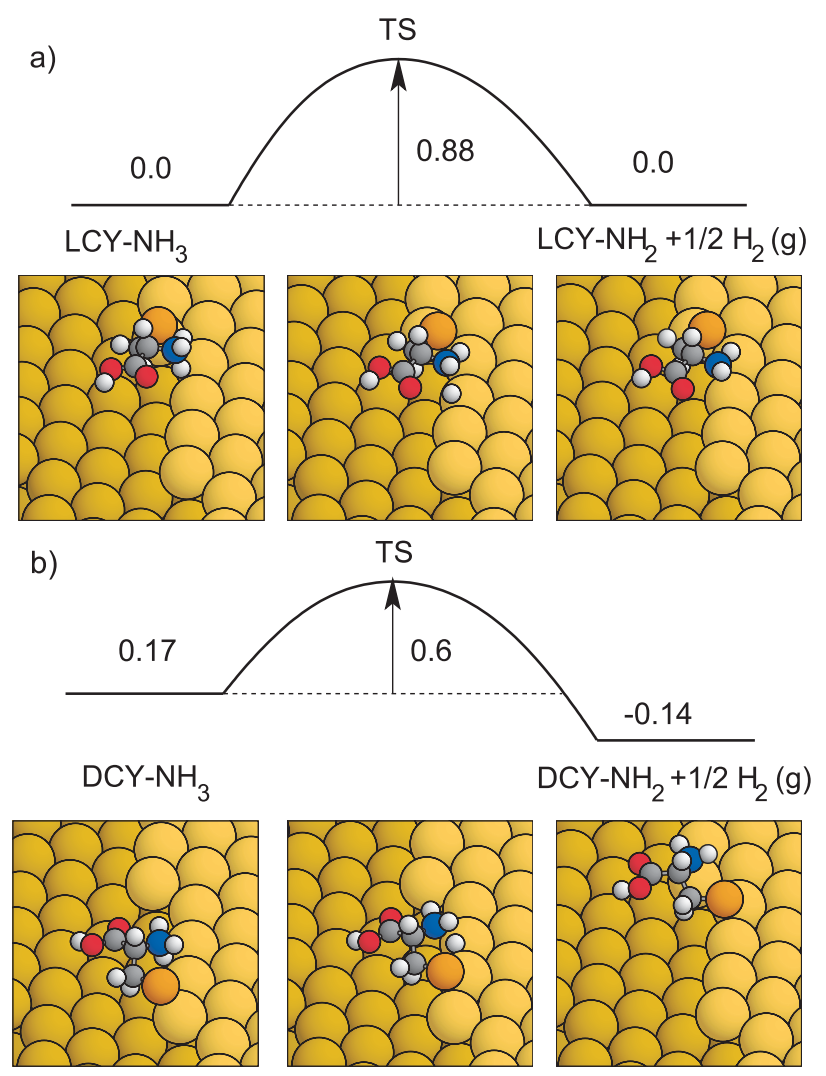

FIG. 4 (color online). Schematic diagram of the total energy of

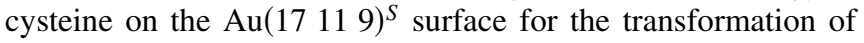
$\mathrm{CY}-\mathrm{NH}_{3}$ to the $\mathrm{CY}-\mathrm{NH}_{2}$ and six reaction snapshots as predicted by DFT. (a) Reaction for $L$-cysteine with a higher activation energy across a transition state (TS) with no net energy gain. (b) Reaction for $D$-cysteine with a lower activation energy and a more favorable $D C Y-\mathrm{NH}_{2}$ total energy. The snapshots show that hydrogen abstraction from $D$-cysteine is accompanied by a directed motion from one kink to the other, while the reaction of $L$-cysteine happens at the same kink. Energies are indicated in $\mathrm{eV}$; the zero line is referred to the $L \mathrm{CY}-\mathrm{NH}_{2} / \mathrm{Au}(17119)$ total energy. Color code as in Fig. 1.

$(0.31 \mathrm{eV})$, which is in line with the absence of $D$-cysteine $\mathrm{NH}_{3}$ after mild annealing of the sample to $\approx 350 \mathrm{~K}$. The six DFT snapshots from the hydrogen abstraction shown in Fig. 4 make further predictions of steric details of the process. While $L$-cysteine reacts on the same kink site, $D$-cysteine moves during hydrogen abstraction in one direction along the step between two kinks.

In conclusion, it is shown that $\mathrm{x}$-ray photoelectron spectroscopy may recover enantiospecific core level binding energies. This proves XPS to be a perfect tool to track the evolution of chemical bonds in enantioselective reactions. The experimental findings were confirmed with density functional theory. Furthermore, theory predicts for the cysteine $/ \mathrm{Au}\left(\begin{array}{lll}17 & 11\end{array}\right)^{S}$ system a higher hydrogen affinity of the $L$-cysteine amino group.

The authors gratefully acknowledge fruitful discussions with Ulrich Heinzmann, Ari Seitsonen, and Jürg
Osterwalder, funding by the Swiss National Science Foundation and the hospitality of the SIM beam line team at the Swiss Light Source. The calculations were performed at the central computational facilities of the École Polytechnique Fédérale de Lausanne and of the Swiss Center for Scientific Computing.

*Electronic address: greber@physik.unizh.ch

[1] R. M. Hazen and D. S. Sholl, Nat. Mater. 2, 367 (2003).

[2] V. Schurig, J. Chromatogr. A 906, 275 (2001).

[3] G. A. Attard, A. Ahmadi, J. Feliu, A. Rodes, E. Herrero, S. Blais, and G. Jerkiewicz, J. Phys. Chem. B 103, 1381 (1999).

[4] A. J. Gellman, J. D. Horvath, and M. T. Buelow, J. Mol. Catal. A: Chem. 167, 3 (2001).

[5] T. Greber, Z. Šljivančanin, R. Schillinger, J. Wider, and B. Hammer, Phys. Rev. Lett. 96, 056103 (2006).

[6] A. Kühnle, T. R. Linderoth, and F. Besenbacher, J. Am. Chem. Soc. 128, 1076 (2006).

[7] A. Ahmadi, G. Attard, J. Feliu, and A. Rodes, Langmuir 15, 2420 (1999).

[8] S. J. Pratt, S. J. Jenkins, and D. A. King, Surf. Sci. 585, L159 (2005).

[9] A. Nielsson, J. Electron Spectrosc. Relat. Phenom. 126, 3 (2002).

[10] R. Fasel, J. Wider, C. Quitmann, K.-H. Ernst, and T. Greber, Angew. Chem., Int. Ed. 43, 2853 (2004).

[11] Y. Zubavichus, O. Fuchs, L. Weinhardt, C. Heske, E. Umbach, J.D. Denlinger, and M. Grunze, Radiat. Res. 161, 346 (2004).

[12] D. Vanderbilt, Phys. Rev. B 41, R7892 (1990).

[13] K. Laasonen, A. Pasquarello, R. Car, C. Lee, and D. Vanderbilt, Phys. Rev. B 47, 10142 (1993).

[14] B. Hammer, L. B. Hansen, and J. K. Nørskov, Phys. Rev. B 59, 7413 (1999).

[15] S. R. Bahn and K. W. Jacobsen, Comput. Sci. Eng. 4, 56 (2002).

[16] E. Pehlke and M. Scheffler, Phys. Rev. Lett. 71, 2338 (1993).

[17] A. Pasquarello, M. S. Hybertsen, and R. Car, Phys. Rev. B 53, 10942 (1996).

[18] G. Gonella, S. Terreni, D. Cvetko, A. Cossaro, L. Mattera, O. Cavalleri, R. Rolandi, A. Morgante, L. Floreano, and M. Canepa, J. Phys. Chem. B 109, 18003 (2005).

[19] A. Kühnle, T.R. Linderoth, B. Hammer, and F. Besenbacher, Nature (London) 415, 891 (2002).

[20] G. Dodero, L. De Michieli, O. Cavalleri, R. Rolandi, L. Oliveri, A. Dacca, and R. Parodi, Colloids Surf. A 175, 121 (2000).

[21] E. Mateo Marti, Ch. Methivier, and C.M. Pradier, Langmuir 20, 10223 (2004).

[22] O. Cavalleri et al., Phys. Chem. Chem. Phys. 6, 4042 (2004).

[23] The coordination of the two gold atoms, which form the bridge site for the thiolate bond are $c_{S}^{L}=6 ; 8$ and $c_{S}^{D}=$ $7 ; 8$, which results in similar total coordinations of 14 and 15 , but not in equal total coordinations of 14 , as stated in Ref. [5]. 